\section{ECCOMAS}

\section{Proceedia}

COMPDYN 2021

$8^{\text {th }}$ ECCOMAS Thematic Conference on Computational Methods in Structural Dynamics and Earthquake Engineering Streamed from Athens, Greece, 28 - 30 June 2021

\title{
NUMERICAL SIMULATIONS OF THE EXPERIMENTAL RESULTS OF STEEL FRAME MODEL WITH ROOF ROLLER SYSTEM SUBJECTED TO BASE EXCIATATIONS
}

\author{
Nikodemos Kouroufexis $^{1}$ and Milton Demosthenous ${ }^{2}$ \\ ${ }^{1}$ Civil Engineer, MSc, Frederick University, Nicosia, Cyprus \\ nikodemoskouroufexis@gmal.com \\ ${ }^{2}$ Dr. Civil Engineer, Professor, Frederick University, Nicosia, Cyprus \\ eng.dm@frederick.ac.cy
}

\begin{abstract}
This paper deals with the experimental and numerical investigation of the effectiveness of a Rolling Mass to the reducing of the resonance response of un experimental and a numerical model of one storey structural system utilizing the shaking table of Frederick University. At the first stage, a sweep test including a range of frequencies of excitations was completed in order to define the resonance response of a steel experimental model simulating one storey building. At the second stage the design and the manufacture of a simple Rolling Mass was completed and its dynamic properties were defined through a sequence of experimental tests with sinusoidal base excitations. At the third stage, the Rolling Mass was attached at the roof of the steel experimental model structure and the influence of that to the resonance response of the model structure was investigated in various cases. Through this investigation the stiffness and the mass ratio of the Rolling Mass system to those of the model structure were defined in order to have the higher level of the reduction of the resonance response. At last but not least, based on the results from the experimental investigation, a numerical model of one storey real structure was designed with or without Rolling Mass at the roof level. This numerical model was subjected also under sinusoidal base excitations in order to study the effectiveness of the Rolling Mass to the resonance response. The normalized results from the experimental investigation of the experimental model structure and those from the numerical investigations of the real model structure were founded out in enough good correlation. The results of this effort are presented and discussed.
\end{abstract}

Keywords: Shaking table, Rolling Mass, Resonance Response 


\section{INTRODUCTION}

During dynamic loading of structures, such as winds, earthquakes or vibrations due to the operation of machines installed on the structures, a resonance response can be occurred, when the frequency of vibration is equal, or almost equal, with the predominant natural frequency of the structure. The level of forces (or accelerations and displacements) generated by resonance vibration are generally too large and usually they don't be acceptable, both for the operation and the safety of the structure. This response is related also with the damping of the structure so as the damping is increased the resonance response is decreased .

The more practical way to reduce the vibration of a structural system is to change its dynamic properties through the strengthening or changing the mass of the system. However, these interventions are very difficult to be applied in many structural systems. In this case, the most effective and economic way to reduce the vibration is to apply an additional dynamic system at a discrete location of the existing structure to change its dynamic properties in order to avoid the resonance. Tuned Mass Dampers (TMDs) belongs into this category of devices. The TMD system is designed taking in the account many parameters and especially the frequency of resonance. It is effective only over a narrow frequency band very close to the resonance frequency. This problem was under investigation in many research efforts $[1,2,3,4]$. The design, manufacture and installation of TMD is a very complicated and expensive procedure. Therefore, for these reasons, today they can have applications only on very specific structures. The sliced mass (SM) and the Rolling Mass (RM) have similar behavior and effects with the TMD. Except of the effectiveness, these specific systems have many differences with the TMD such as the manufacture procedure, the installation on the structures, the cost as well the design and the control of their response. On the other hand, the sliced mass (SM) and the rolling mass (RM) can easily be manufactured and installed on a structure but very difficult to be controlled in the way for the reduction of the resonance response.

This paper deals with the experimental and numerical investigation of the effectiveness of a Rolling Mass (RM) to the reducing of the resonance response of un experimental [5] and a numerical model of one storey structural system. For the purposes of this effort the shaking table of Frederick University was used.

- At the first stage, a sweep test including a range of frequencies of excitations was completed in order to define the resonance response of a steel experimental model simulating one storey building.

- At the second stage the design and the manufacture of a simple Rolling Mass (RM) was completed and its dynamic properties were defined through a sequence of experimental tests with sinusoidal base excitations.

- At the third stage, the RM was attached at the roof of the steel experimental model structure and the influence of that to the resonance response of the model structure was investigated in various cases. Through this investigation the stiffness and the mass ratio of the RM to those of the model structure were defined in order to have the higher level of the reduction of the resonance response.

- At last but not least, based on the results from the experimental investigation, a numerical model of one storey real structure was designed with or without Rolling Mass (RM) at the roof level. This numerical model was subjected also under sinusoidal base excitations in order to study the effectiveness of the RM to the resonance response. The normalized results from the experimental investigation of the experimental model structure and those from the numerical investigations of the real model structure were founded out in enough good correlation. 


\section{EXPERIMENTAL INVESTIGATION}

\subsection{Experimental model structure and results from sweep test}

For the purpose of this investigation, a flexible steel frame system in small scale was used consisted by two flexible vertical elements and a rigid slab. From this point of view we can assume that this model represent a Single Degree of Freedom (SDOF) system. Before any test, the mass and the stiffness of this model were measured. This model was fixed at the platform of the shaking table and was subjected on a range of frequencies of excitations $(1.5-8.5 \mathrm{~Hz})$ with step $0.5 \mathrm{~Hz}$, including of course in this range of frequencies the predominant frequency of this steel frame. In all the cases the max. base acceleration was kept constant $\left(2.0 \mathrm{~m} / \mathrm{sec}^{2}\right)$. The base acceleration as well the acceleration response of the experimental model were measured in time domain and restored using a data acquisition system (figure 1). Through this sequence of tests (well known as sweep test) the resonance response of the SDOF system was defined as well the predominant frequency and the viscous damping of the experimental model. The results of the resonance response from this test are summarized in figure 2.

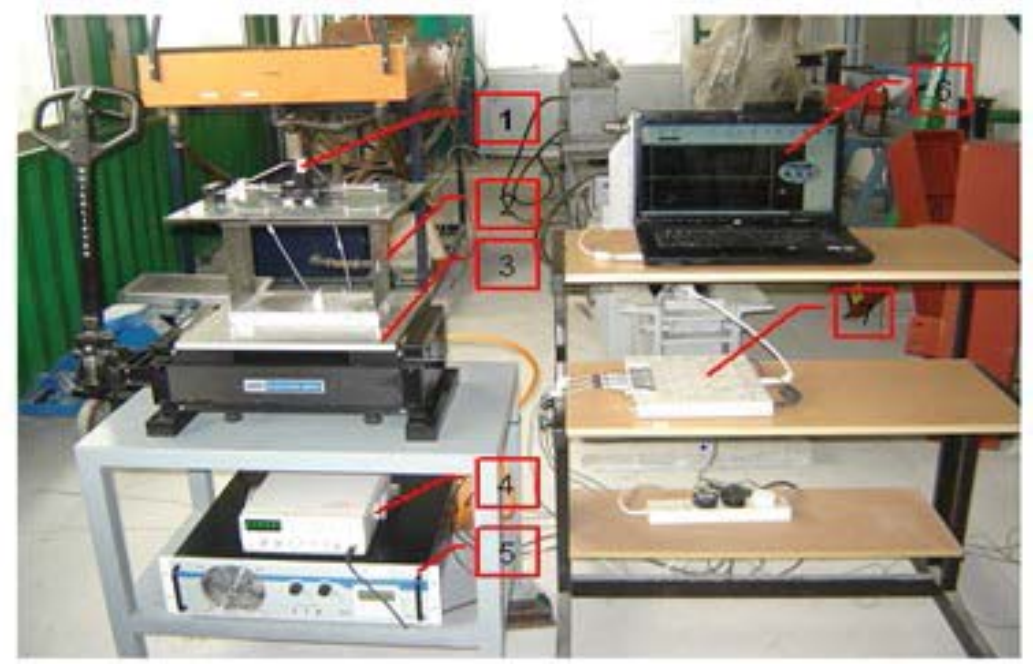

Figure 1: The experimental setup with the model structure

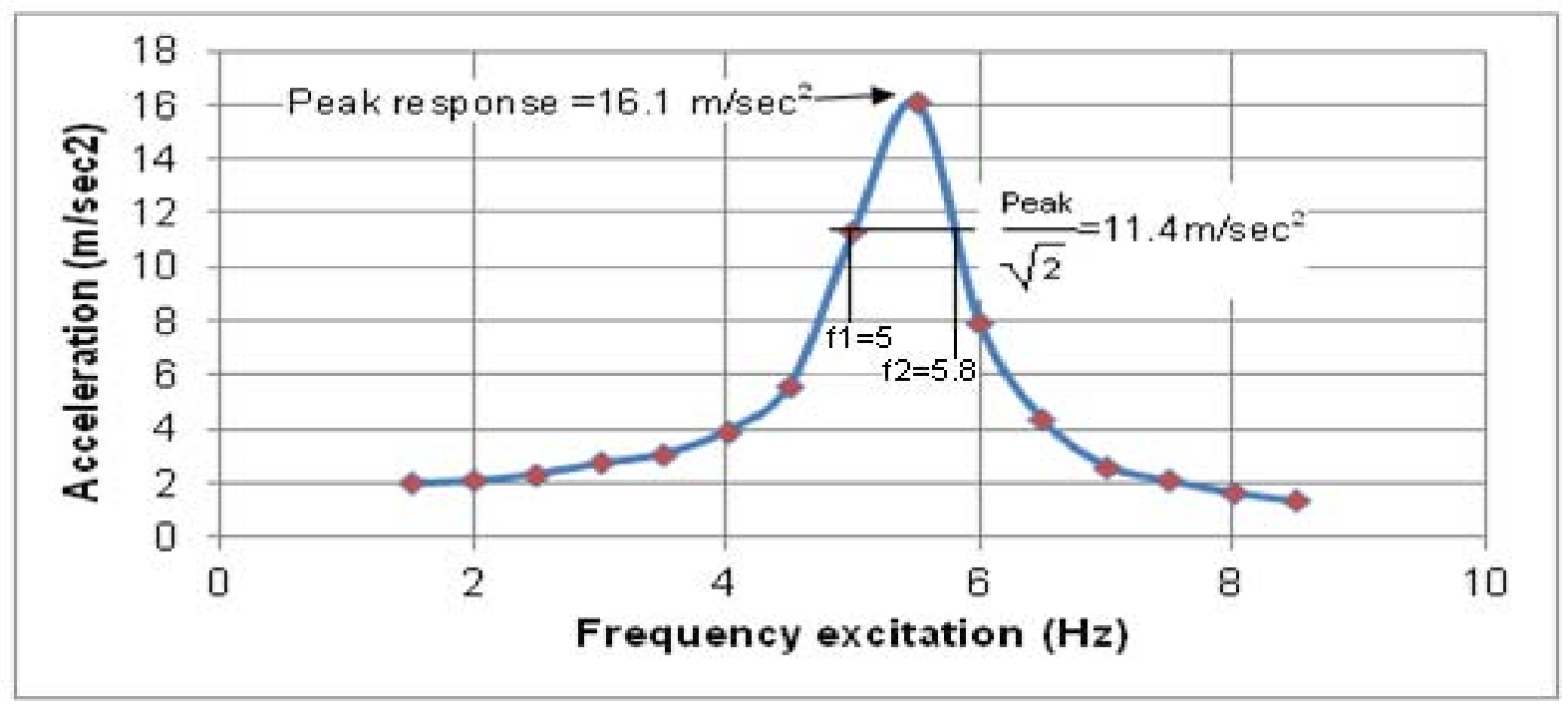

Figure 2: The resonance response of the experimental model 


\subsection{Experimental Rolling Mass (RM) and results from sweep test}

At the second stage the design and the manufacture of a single Rolling Mass (RM) was completed. It is a symmetrical system with four rollers, free standing in a metallic plate and able to roll in horizontal direction. At the edge of this plate two rigid metallic systems were fixed. The rolling mass was connected with these rigid metallic systems with springs arranged in symmetrical configuration (figure 3). This system was attached at the platform of the shaking table and was subjected to a range of frequencies of excitations with constant max. base acceleration but with various values of mass of the RM and various values of stiffness of the springs. Before any test the stiffness of the used springs were measured. For each test, the base acceleration as well the response acceleration of the RM were measured and restored. Using these data, the resonance response of the RM, the predominant frequency and the viscous damping were estimated. The results from one case from this sequence of tests are presented in figure 4.

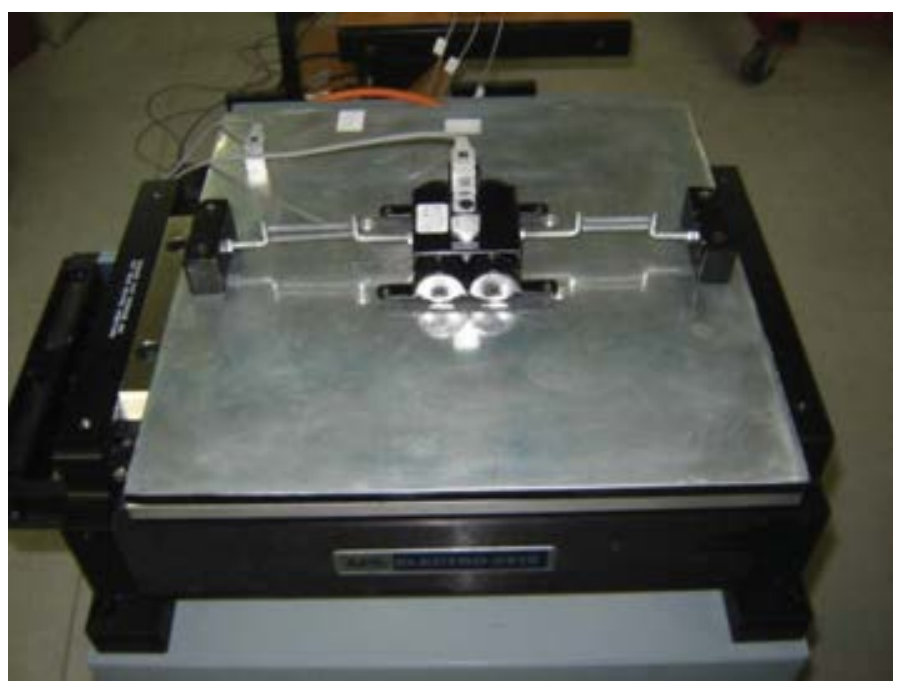

Figure 3: The rolling mass on the shaking table

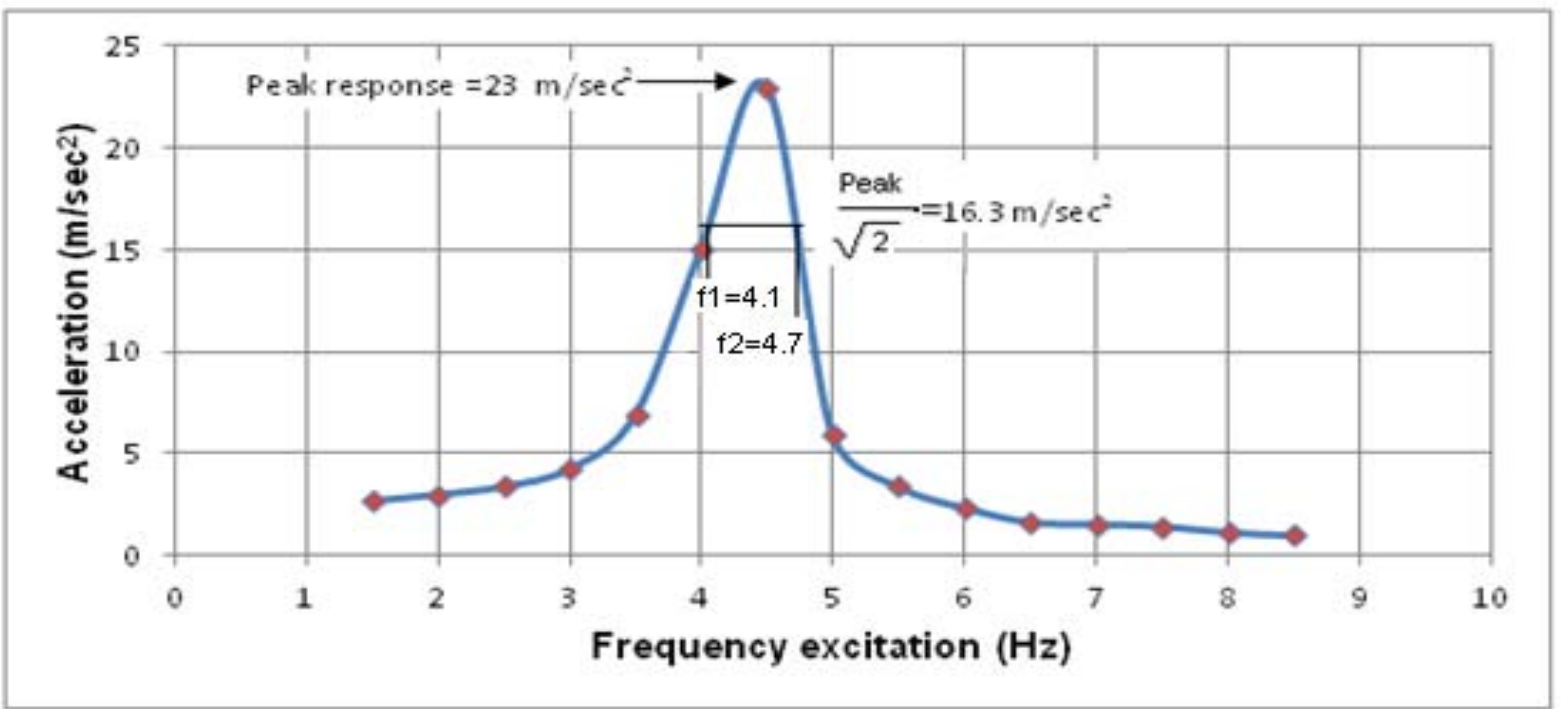

Figure 4: Resonance response of the Rolling Mass (RM) 


\subsection{Experimental model structure with RM and results from sweep test}

At the third stage, the system with the RM was attached at the roof of the model structure and was subjected in a large number of sinusoidal base excitations using the same range of frequencies and max. base acceleration as in the previous tests (figure 5). The results from a test are presented in figure 6 and correlated with those from the resonance response of the experimental model without the RM. As can be seen the response of the experimental model with RM is reduced drastically in a narrow band of frequencies closed to the resonance frequency (figure 6). However, two other peaks of the response of this system with RM are occurred but with lower level of amplitudes, one at the left of the resonance frequency with frequency F1 and one at the right with frequency F2. The left one represent the case with inphase response of the RM with that of the model structure and the right one the case of the out-of-phase response.

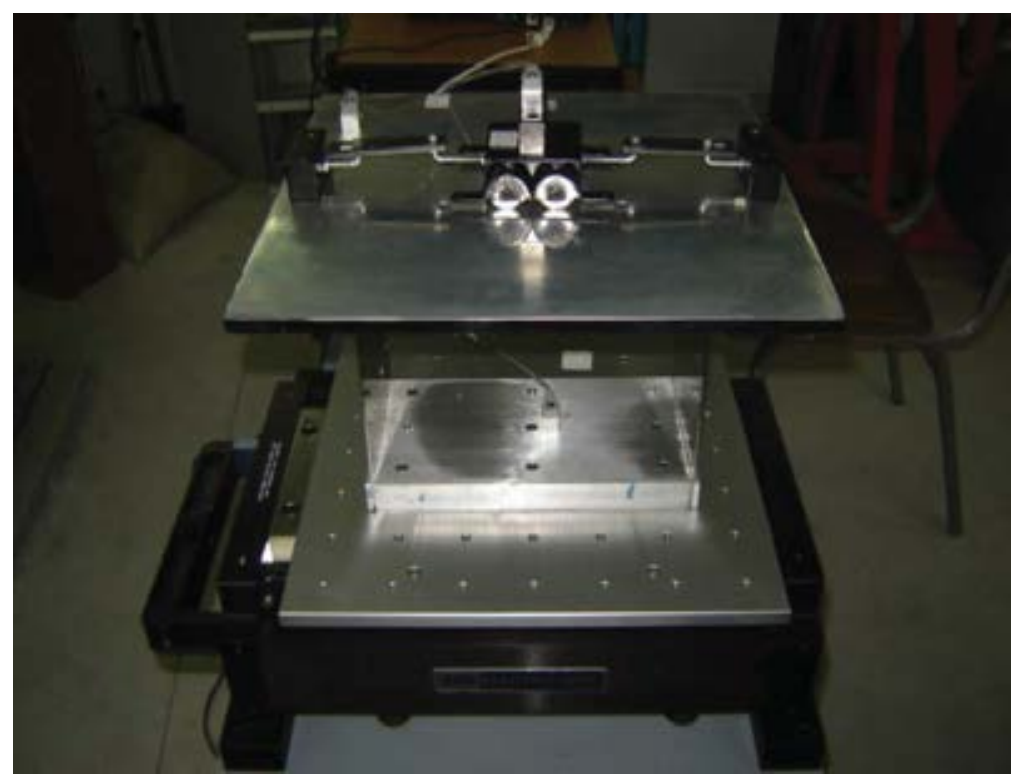

Figure 5: The experimental model with the RM on the shaking table

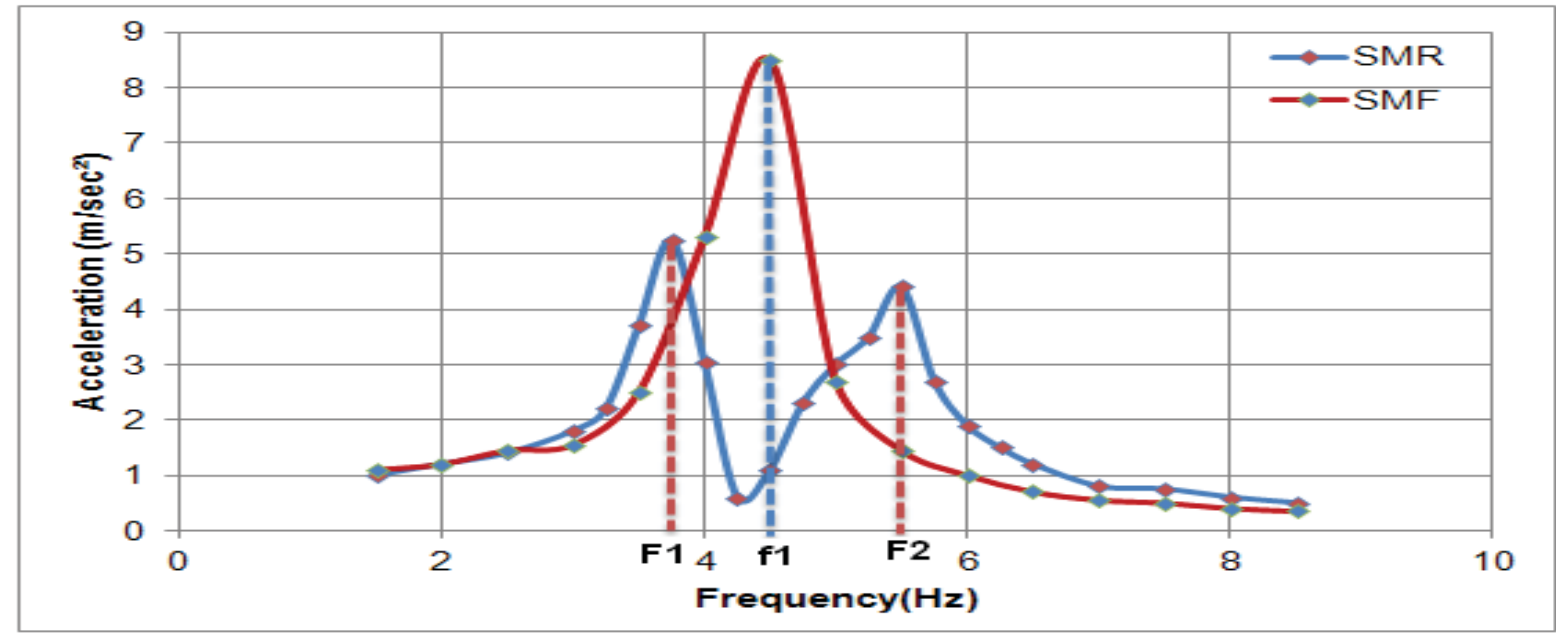

Figure 6: Correlation of the resonance response of the experimental model without the RM (SMF) with the response of the same model with the RM (SMR) 


\subsection{Results from experimental parametric investigations}

An extended parametric investigation with 13 sweep tests were completed in order to examine the influence of the mass and the predominant frequency of the RM to the response of the model structure. In the first case, the mass ratio $(\mathrm{k}=$ mass of $\mathrm{RM} /$ mass of model structure) was kept constant $(\mathrm{k}=16 \%)$ but the frequency of RM was changed from test to test $(\delta=$ natural frequency of RM / natural frequency of model structure) using different springs. Seven sweep tests were completed in the same range of frequencies and constant max. base acceleration as before. The results from this investigation are presented in figure 7. As can be seen, the response of the model structure with RM is changed drastically form test to test. In the second case, the ratio of frequencies was kept constant and equal to one $(\delta=1)$ and the ratio of mass $(\mathrm{k})$ was changed from test to test fixing additional masses on the RM. The results from six sweep tests are presented in figure 8 . As can be seen, in his case the response of the model structure with RM is more constant. However, it was founded out that the lower amplitude of the response is occurred in the case when the ratio of the mass of the RM over the mass of the model structure is $10 \%(\mathrm{k}=10 \%)$.

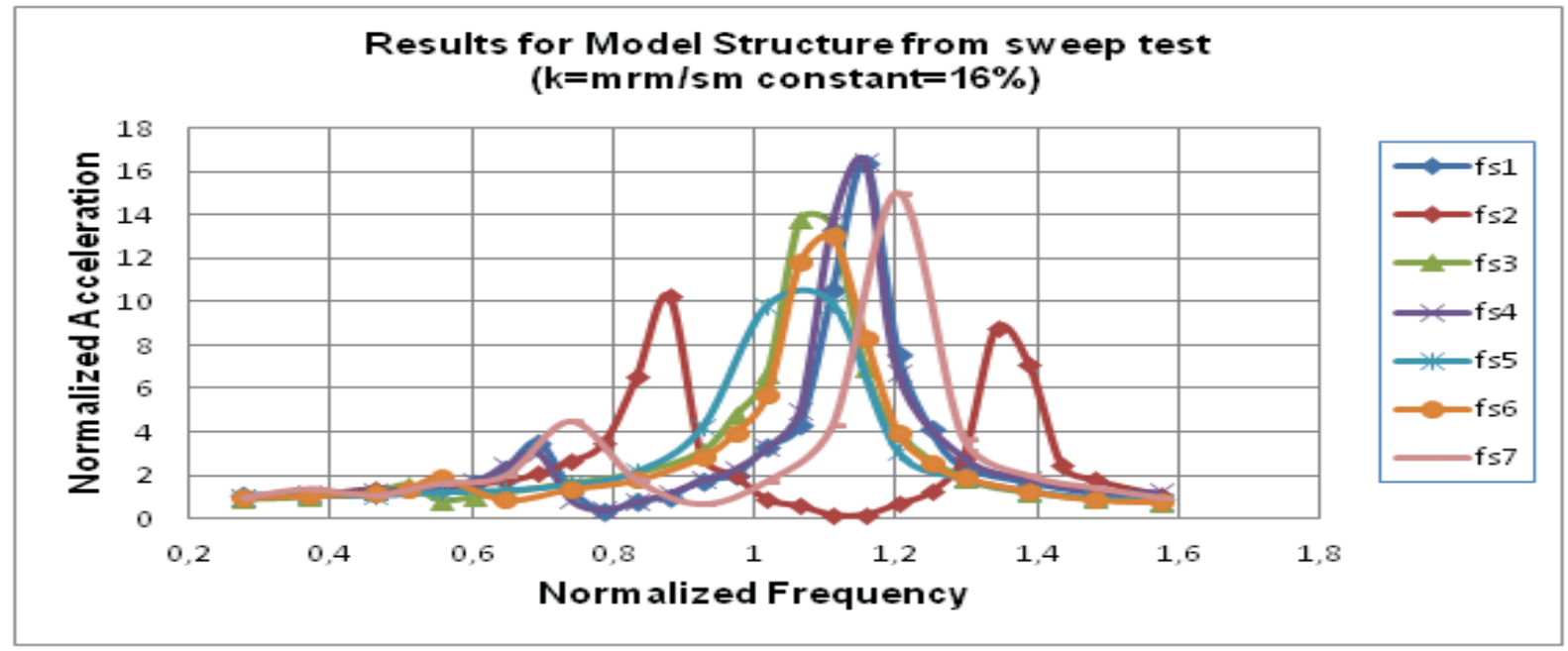

Figure 7: Experimental results of the experimental model with RM from seven sweep tests with constant ratio of masses $(\mathrm{k}=16 \%)$

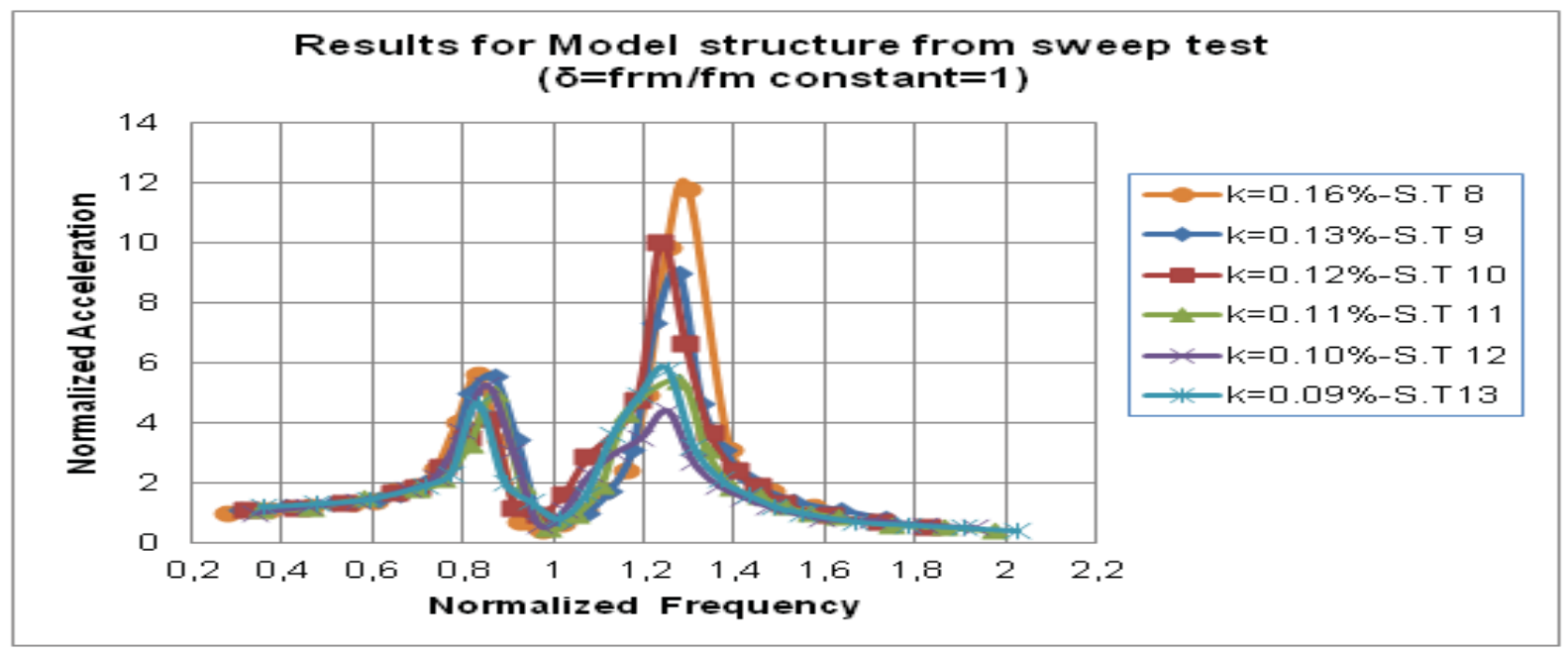

Figure 8: Experimental results of the experimental model with RM from six sweep tests with constant ratio of frequencies $(\delta=1)$ 


\subsection{Prediction of the fundamental frequencies of experimental model with RM}

At first, the assumption that the experimental model without the RM is a Single Degree of Freedom (SDOF) system is adopted. Therefore, the case of the experimental model with RM assumed that is a Two Degree of Freedom (2DOF) system. Using analytical methods from structural dynamic theories, the prediction of the fundamental frequencies of the experimental model with RM (F1, F2) and the difference between these two frequencies (F2-F1) are estimated. The F1 corresponds to the 1st mode with the in-phase response of the model structure and the RM and the F2 corresponds to 2nd mode with out-of-phase response. The predicted results from the analysis of 13 experimental sweep tests are presented in figures 9 and correlated with those which measured during the experimental tests. As can be seen from the diagrams of this figure, there are enough good correlation between experimental results and the predicted values. These observations allowed us to continue the investigation of this problem through numerical simulations as will be presented in the follow section.

1st mode (F1)

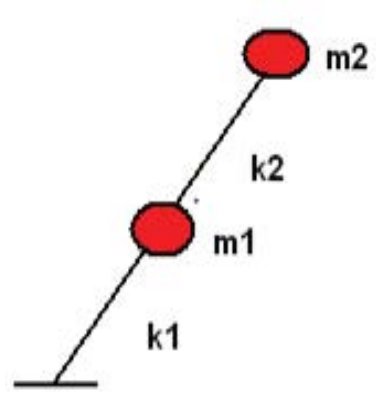

2nd mode (F2)

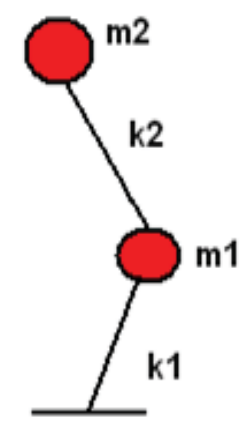

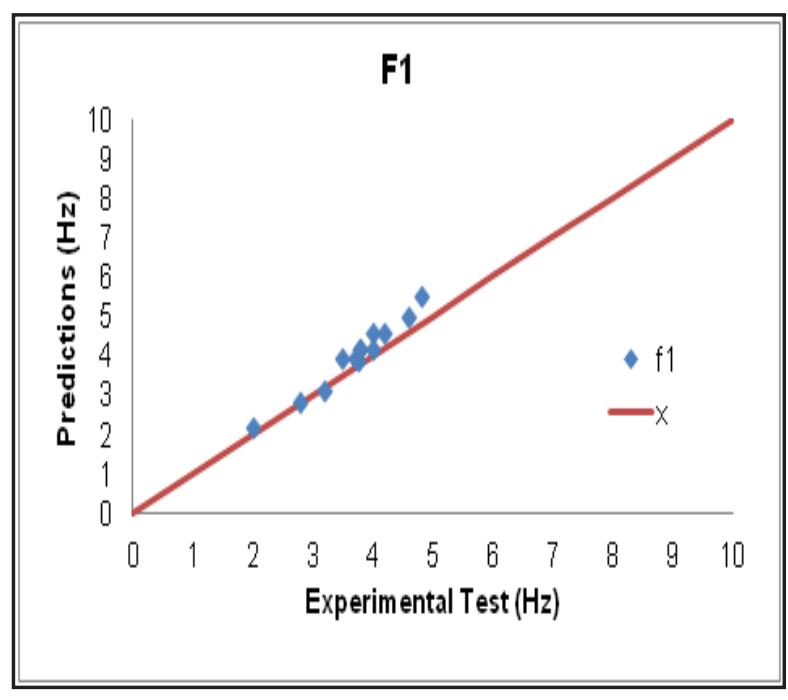

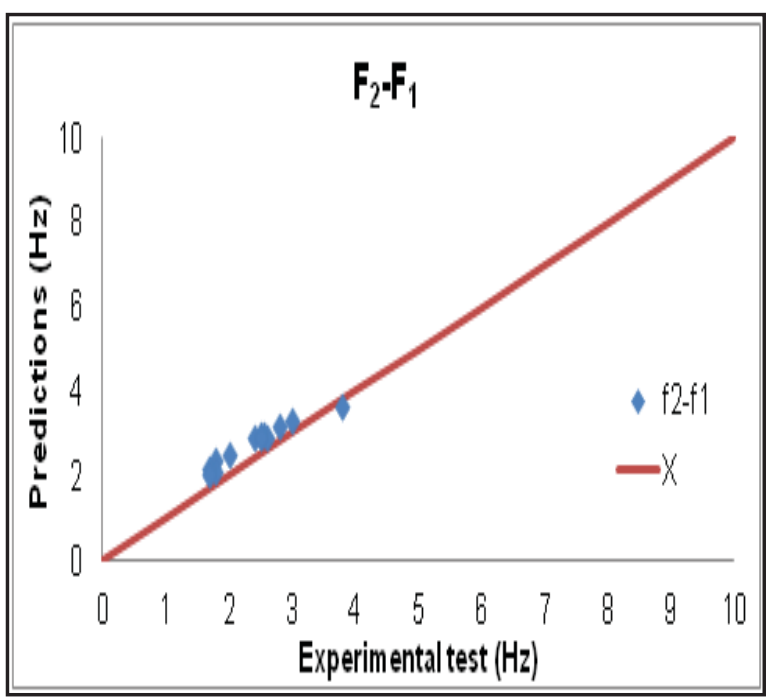

Figure 9: Prediction of fundamental frequencies of the experimental model with RM and correlation with the experimental result 


\section{NUMERICAL INVESTIGATION}

\subsection{Description of the numerical model}

Based on the results from the experimental investigation, a numerical model of one storey frame of a real structure with height 3.0m was designed with or without Rolling Mass (RM) at the roof level. This structural model is consisted by two columns and an horizontal rigid beam. At the first stage, through a modal analysis the predominant frequency of the structural system without the RM was founded out ( $\mathrm{fo}=2.85 \mathrm{~Hz}$ ). The two columns are expanded over the beam as rigid elements. The mass of the RM was defined to be equal to $10 \%$ of the mass of the model of the real structure and to be very stiff using horizontal and diagonal bracers. The attach of the RM at the middle of the beam was done through specific supports. The restrictions of these supports were defined in order to allow the horizontal displacement in two horizontal directions but without creation of bending moments, simulating the roller supports of the experimental model. Using horizontal springs, the RM was connected with the rigid elements over the columns in both directions (figure 10). Through an extended numerical investigation the characteristics of the horizontal springs as well those of the specific supports were defined with the main scope the predominant frequency of the RM to be equal to the predominant frequency of the numerical model of the frame structure $(2.85 \mathrm{~Hz})$. This model structure with RM was subjected in a sweep test for various frequencies keeping constant the max. base acceleration. The shape of the used sinusoidal signal was similar to that developed by the shaking table as it is presented in figure 11 .

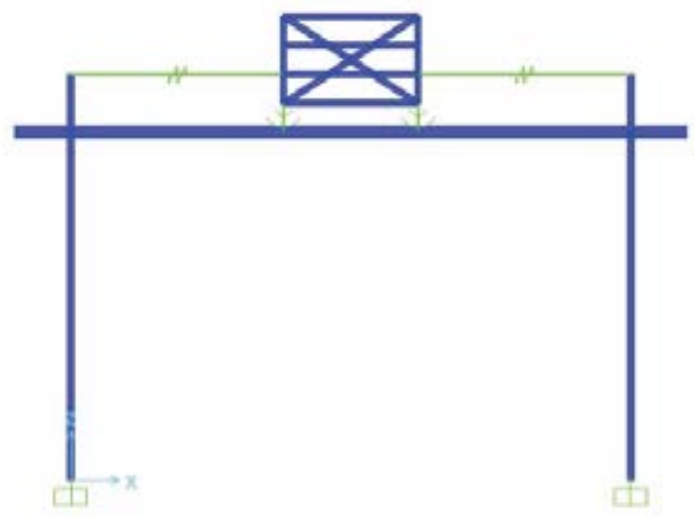

Figure 10: Numerical model of frame system with the RM at the roof

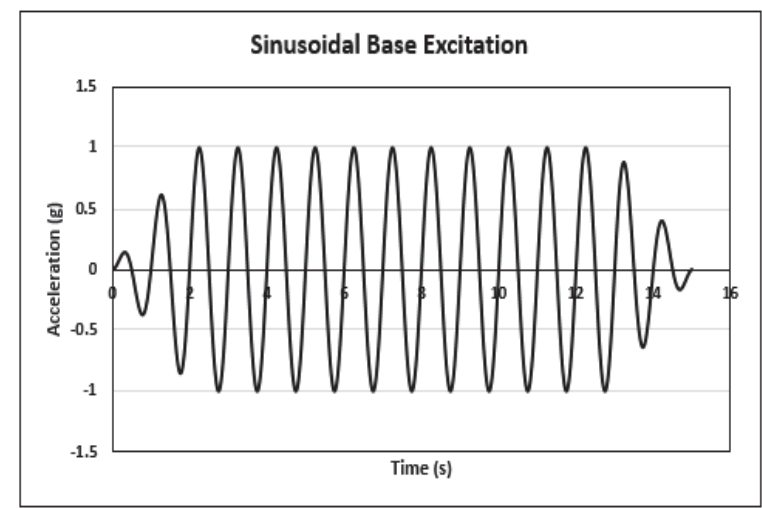

Figure 11: Shape of the sinusoidal base acceleration

\subsection{Modal and step by step time history analysis}

An extended modal analysis of the numerical models was completed to define the predominant modes of them because the final design of the numerical model with RM is based on the modal characteristics, both the structural system and the RM. As has been mentioned above, at the first stage the predominant frequency of the frame structure without the RM was founded out. At the second stage the characteristics of the used springs and the those of the supports of the RM on the rigid beam were defined with the main scope the fundamental frequency of the RM to be equal to that of the frame system. Finally, the predominant modes of the frame system with the RM were estimated using SAP2000. The first two mode shapes of this model are presented in figure 12 giving also the corresponding values of frequencies. As can be seen, the 1st mode describes an in-phase response of the structural system with that of the $\mathrm{RM}$ with $\mathrm{f} 1=2.43 \mathrm{~Hz}$ and the 2 nd mode an out-of-phase response with $\mathrm{f} 2=3.45 \mathrm{~Hz}$. 
After that, a step by step time history analysis of the structural system with RM was completed using as time history of the base acceleration the shape which was presented in figure 11 and for various values of frequencies and max. base accelerations. The results from two cases are presented in figures $13 \& 14$. In the first case (figure 13) the frequency excitation was equal to the fundamental frequency of the 1 st mode, $\mathrm{fl}=2.43 \mathrm{~Hz}$. The black line correspond to the base acceleration (excitation), the red one to the acceleration response of the frame system and the blue one to the acceleration response of the RM. As can be seen from this figure, there is in-phase response of the frame system with the RM however, the max. acceleration of the RM is much higher than that of the frame system. In the second case (figure 14) the frequency excitation was equal to the fundamental frequency of the 2 nd mode, $\mathrm{f} 2=3.45 \mathrm{~Hz}$. In this case, out-of-phase response is occurred between the RM and the frame system but also in this case the max. acceleration of the RM is much higher than that of the frame system. All these observations are in a good agreement with those from the experimental investigation.
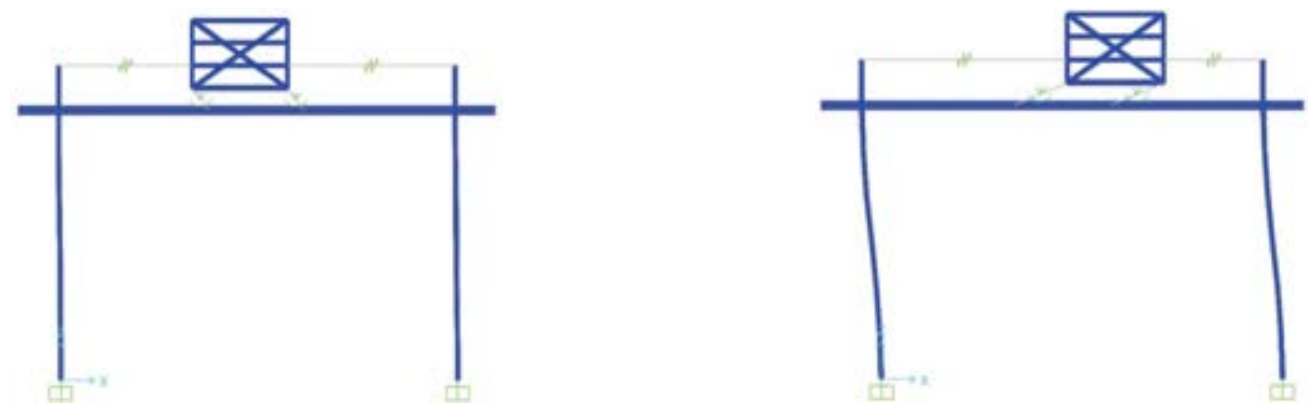

Figure 12: Mode shapes of 1 st mode $(\mathrm{f} 1=2.43 \mathrm{HZ})$ and $2 \mathrm{nd}$ mode $(\mathrm{f} 2=3.45 \mathrm{~Hz})$ of the structural model with $\mathrm{RM}$

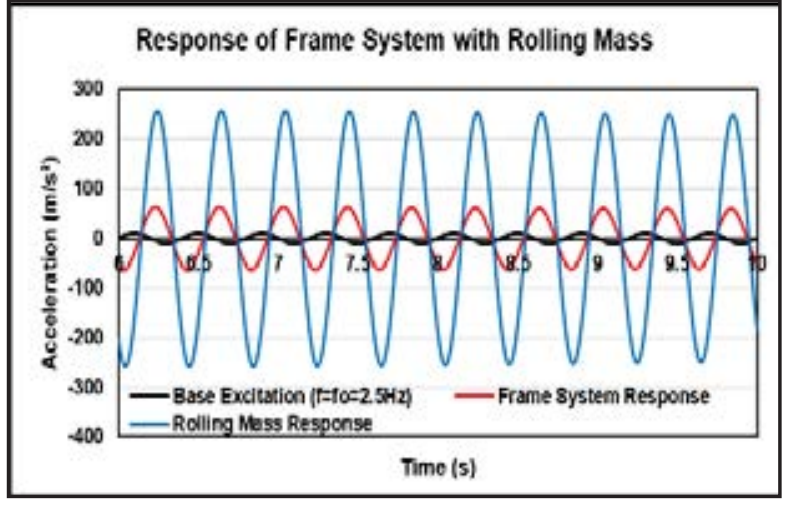

Figure 13: In-phase response - freq. ex. f1=2.43HZ

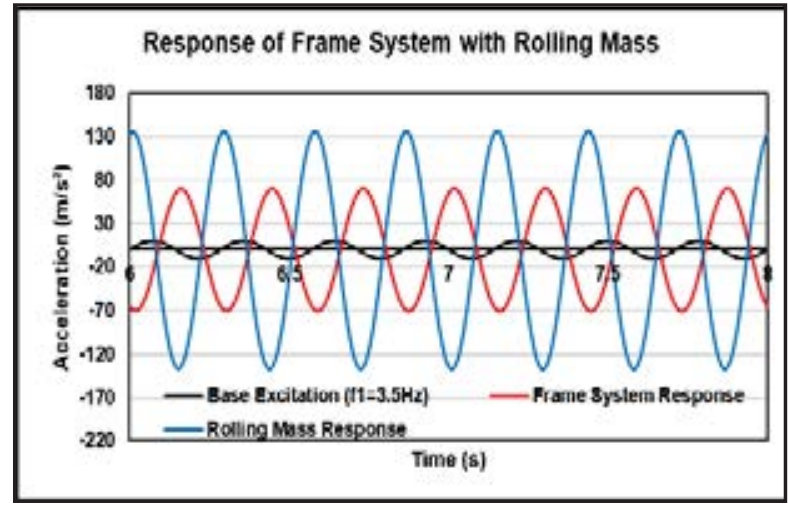

Figure 14: Out-of-phase response- freq. ex. f1=3.45HZ

\subsection{Results for sweep analysis and correlation with experimental results}

The numerical models of the frame structure with and without RM attached at the roof beam were subjected in a sweep analysis in a range of frequencies of excitation and constant max. base acceleration. The results from these analyses are summarized in figure 15 in normalized form (response acceleration / resonance max. acceleration response) together with the corresponding normalized results of the experimental model from the sweep tests. As can be seen from this figure there is a good agreement between numerical and experimental results. Some differences between the results of the two models are under investigation. 


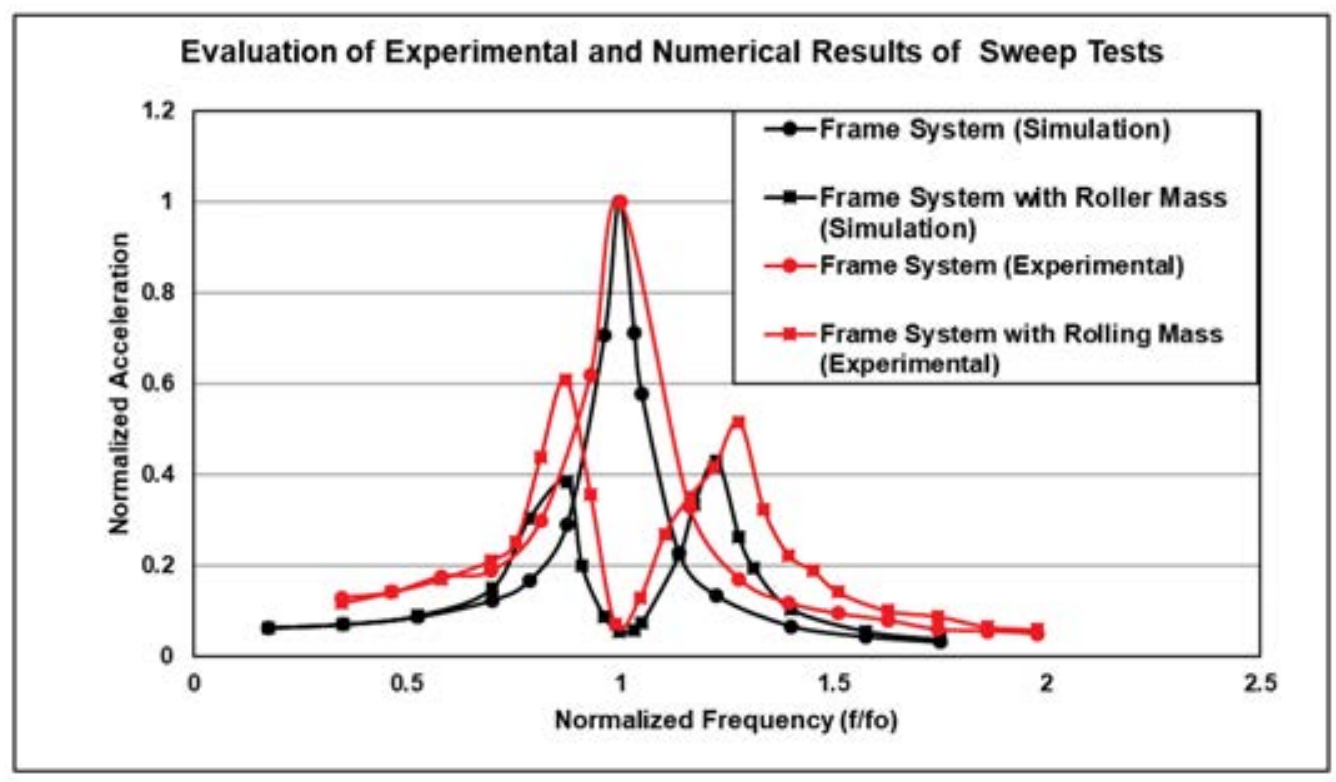

Figure 15: Correlation of numerical and experimental results

\section{CONCLUSIONS}

Through the experimental and numerical investigation of this paper, the follow conclusions can be made:

- During the experimental investigation it was founded out that the Rolling Mass (RM) can be used in experimental models in order to reduce the resonance response.

- The reduction of the resonance response becomes higher when the mass ratio (mass of the $\mathrm{RM}$ / mass of the structure) is equal to $10 \%$ and the frequency of the rolling mass is equal to that of the structure.

- In phase and out-of-phase response of RM to that of the frame structure can develop but in both cases the response is much lower than that developed during resonant response of the structure without the RM.

- A good correlation was observed between numerical and experimental normalized results, therefore similar conclusions can be made also from the numerical investigation

\section{REFERENCES}

[1] Haga.Y. ed al. (1992). Tuned mass Damper for long-period buildings. Tsukuba, p.p.2311-2317.

[2] Winkel, B. Keith. L. (2008). Peak-Frequency Responses and Tuned Mass Dampers Exciting Applications of Systems of Differential Equations. UMAP. p30.

[3] Chey, M-H. ed al. (2009). Energy-dissipative Semi-Active Tuned Mass Damper Building Systems for Structural Damage Reduction. p15.

[4] Liedes, T. (2009). Improving the performance of the semi-active tuned mass damper. Oulu Finland: University of Oulu. p159

[5] Theodosiou, A. (2012). Experimental investigation of the dynamic response of model structures with active rolling mass, Final Year Project, Department of Civil Engineering, Frederick University, Supervisor, Prof. Milton Demosthenous 